TRANSACTIONS OF THE

AMERICAN MATHEMATICAL SOCIETY

Volume 349, Number 10, October 1997, Pages 4251-4264

S $0002-9947(97) 01772-8$

\title{
TAME COMBINGS OF GROUPS
}

\author{
MICHAEL L. MIHALIK AND STEVEN T. TSCHANTZ
}

\begin{abstract}
In this paper, we introduce the idea of tame combings for finitely presented groups. If $M$ is a closed irreducible 3-manifold and $\pi_{1}(M)$ is tame combable, then the universal cover of $M$ is homeomorphic to $\mathbb{R}^{3}$. We show that all asynchronously automatic and all semihyperbolic groups are tame combable.
\end{abstract}

\section{INTRODUCTION}

In [9], V. Poénaru proved that if a closed irreducible 3-manifold $M$ has an almost convex fundamental group, then the universal cover of $M$ is homeomorphic to $\mathbb{R}^{3}$. A. Casson has devised a group theoretic condition that implies certain closed irreducible 3-manifolds are covered by $\mathbb{R}^{3}$. In particular, Casson's ideas can be used to show that a closed irreducible 3-manifold with infinite word hyperbolic fundamental group is covered by $\mathbb{R}^{3}$. This result is also obtained in [2] using different techniques. In this paper, we develop the notion of a tame combing for finitely presented groups, and show that all asynchronously automatic and all semihyperbolic groups are tame combable. If a closed irreducible 3-manifold $M$ has tame combable fundamental group, then we show that the universal cover of $M$ is homeomorphic to $\mathbb{R}^{3}$. The main theorem of [9] easily follows from our Theorems 1 and 2. (See the remark following Theorem 2 for more on this.) In [3], Brick and Mihalik examine an idea related to Casson's work, the quasi-simply-filtered (QSF) property, which can also be used to show certain 3 -manifolds are covered by $\mathbb{R}^{3}$. Our Theorem 3 states that any tame combable group is QSF.

For $G$ a finitely presented group, $X$ any compact polyhedron with $\pi_{1}(X)=G$, and $\tilde{X}$ the universal cover of $X$, our combing condition on $G$ is equivalent to the following geometric property for $\tilde{X}$ : If $C$ is a finite connected subcomplex of $\tilde{X}$, then $\pi_{1}(\tilde{X}-C)$ is finitely generated. In [10], T. Tucker shows that if $M$ is a non-compact $P^{2}$-irreducible 3-manifold, and for each finite subcomplex $C$ of $M$, $\pi_{1}(M-C)$ is finitely generated, then $M$ is a missing boundary manifold (i.e., there exist a compact 3-manifold $N$ and a closed subset $K$ of the boundary of $N$ such that $N-K$ is homeomorphic to $M$ ). If, additionally, $M$ is the universal cover of a closed 3-manifold, then results in [5] imply that $M$ is homeomorphic to $\mathbb{R}^{3}$ (bypassing the QSF theory).

For technical reasons, the CW-complexes considered in [3] are those where attaching maps on 2-cells are piecewise linear (PL). For the most part we are interested in polyhedra, since all of our applications are directed toward the (covering)

Received by the editors July 11, 1995 and, in revised form, March 22, 1996.

1991 Mathematics Subject Classification. Primary 20F05; Secondary 57M20.

(C)1997 American Mathematical Society 
conjecture - All closed irreducible 3-manifolds with infinite fundamental group are covered by $\mathbb{R}^{3}$.

The following definition is due to Stephen Brick.

Definition. A finitely presented group $G$ is quasi-simply-filtered (QSF) if for some (equivalently any) finite CW-complex $X$ with $\pi_{1}(X)=G$, the universal cover $\tilde{X}$ of $X$ has the following property: If $C$ is a finite, connected subcomplex of $\tilde{X}$, then there exist a finite, simply connected complex $K$ and a cellular map $f: K \rightarrow \tilde{X}$ such that $\left.f\right|_{f^{-1}(C)}$ is a homeomorphism of $f^{-1}(C)$ onto $C$.

The notion of bounded and asynchronously bounded combings of groups has gained notoriety in connection with the study of automatic and asynchronously automatic groups (see [6]).

Definition. Suppose $X$ is a 1-complex with fixed basepoint $*$ and edge path metric d. A discrete path in $X$ is a map $p:\left[0, T_{p}\right] \cap \mathbb{N} \rightarrow X^{0}$, where $T_{p} \in \mathbb{N}$ is the length of $p$, such that $d(p(t), p(t+1)) \leq 1$ for all $t<T_{p}$. For simplicity, if $p$ is a discrete path and $t>T_{p}$, interpret $p(t)$ to be $p\left(T_{p}\right)$. A combing of $X$ is a map $\Psi$ which assigns to each $x \in X^{0}$ a discrete path $p(t)=\Psi(x, t)$ such that $p(0)=*$ and $p\left(T_{p}\right)=x$. A combing $\Psi$ of $X$ is bounded if there exists a constant $K$ such that, for all adjacent $x, y \in X^{0}$ and all $t, d(\Psi(x, t), \Psi(y, t))<K$. A combing $\Psi$ of $X$ is asynchronously bounded if there exists a constant $K$ such that, for all adjacent $x, y \in X^{0}$, there exist non-decreasing surjections $\alpha, \beta: \mathbb{N} \rightarrow \mathbb{N}$ such that, for all $t, d(\Psi(x, \alpha(t)), \Psi(y, \beta(t)))<K$. A finitely presented group $G$ is said to have a bounded or asynchronously bounded combing if there exists a bounded or asynchronously bounded combing of the 1-skeleton of the universal cover of some (equivalently any) finite complex $X$ with $\pi_{1}(X) \cong G$.

The definition of combings in terms of discrete paths proves to be somewhat inconvenient for our purposes. We offer the obvious reformulation of combings in terms of continuous paths and a crucial generalization of this notion.

Definition. Suppose $X$ is a 2-complex. A 0-combing of $X$ is a homotopy $\Psi$ : $X^{0} \times[0,1] \rightarrow X^{1}$ such that $\Psi(x, 1)=x$, for all $x \in X^{0}$, and $\left.\Psi\right|_{X^{0} \times\{0\}}$ is constant. A 1-combing of $X$ is a homotopy $\Psi: X^{1} \times[0,1] \rightarrow X$ such that $\Psi(x, 1)=x$, for all $x \in X^{1},\left.\Psi\right|_{X^{1} \times\{0\}}$ is constant, and $\operatorname{im}\left(\left.\Psi\right|_{X^{0} \times[0,1]}\right) \subseteq X^{1}$ (i.e., so $\left.\Psi\right|_{X^{0} \times[0,1]}$ is a 0 -combing). A 0 -combing $\Psi$ of $X$ is bounded if there exists $K>0$ such that for all adjacent $x, y \in X^{0}$, there are orientation preserving homeomorphisms $\alpha, \beta:[0,1] \rightarrow[0,1]$ such that $d(\Psi(x, \alpha(t)), \Psi(y, \beta(t))) \leq K$ for all $t \in[0,1]$.

Clearly, any combing can be made into a 0-combing by connecting successive vertices in a combing path by edges and parameterizing. Conversely, a 0 -combing gives rise to a combing by taking the sequence of (successively distinct) vertices in each 0-combing path. Moreover, an asynchronously bounded combing will correspond to a bounded 0-combing (we omit the term "asynchronously" to simplify our terminology and note that 0 -combing paths are all parameterized by $[0,1]$ ). A 1-combing contains homotopies showing that the loop formed by any edge $e$ and the 0 -combing paths to the endpoints of $e$ is homotopically trivial. Hence, while any connected 2-complex is 0 -combable, a connected 2-complex will be 1-combable iff it is also simply connected. One can also give an analogous definition of what it would mean for a complex to be 2-combable, but this notion would only become interesting in considering complexes of dimension 3 or higher, since a 2-complex is (essentially by definition) 2 -combable iff it is contractible. 
Definition. If $\Psi$ is a 0 -combing of $X$, then $\Psi$ is tame if for each compact set $C \subseteq X$ there exists a compact set $D \subseteq X$ such that, for all $x \in X^{0}, \Psi^{-1}(C) \cap(\{x\} \times[0,1])$ is contained in one path component of $\Psi^{-1}(D) \cap(\{x\} \times[0,1])$. If $\Psi$ is a 1-combing of $X$, then $\Psi$ is tame if $\left.\Psi\right|_{X^{0} \times[0,1]}$ is a tame 0-combing and, for each compact set $C \subseteq X$, there exists a compact set $D \subseteq X$ such that, for each edge $e$ of $X$, $\Psi^{-1}(C) \cap(e \times[0,1])$ is contained in one path component of $\Psi^{-1}(D) \cap(e \times[0,1])$. (Observe that if $E$ is a compact subset of $X$ containing a $D$ as above, then one path component of $\Psi^{-1}(E)$ contains $\Psi^{-1}(C)$ as well; hence it suffices to take $D$ sufficiently large. Furthermore, if the condition is satisfied for a compact $C^{\prime} \supseteq C$ then it will also be satisfied for $C$, so we need only that the condition holds for all sufficiently large $C$. Thus we could have taken the compact sets above to be subcomplexes.)

We now list our results.

Theorem 1. If $X$ and $Y$ are finite, connected 2-dimensional $C W$-complexes and $\pi_{1}(X) \cong \pi_{1}(Y)$, then the universal cover of $X$ has a tame 0-combing or tame 1-combing iff the universal cover of $Y$ does.

Definition. A finitely presented group $G$ has a tame 0-combing (resp. 1-combing) if for some (equivalently any) finite 2-dimensional CW-complex $X$ with $\pi_{1}(X) \cong G$, the universal cover of $X$ has a tame 0 -combing (resp. 1-combing).

Theorem 2. Let $\tilde{X}$ be the universal cover of a finite 2-dimensional polyhedra $X$. Then $\tilde{X}$ has a tame 1-combing iff, for each finite subcomplex $C \subseteq \tilde{X}, \pi_{1}(\tilde{X}-C)$ is finitely generated (i.e., each component of $\tilde{X}-C$ has finitely generated fundamental group).

Remark. If $C$ is a finite set of generators for a group $G$, the Cayley graph $\Gamma(G, C)$ of $G$ with respect to $C$ is the directed labeled graph with vertex set $G$, and with a directed edge with label $e$ from $g$ to $g e$ for each $g \in G$ and $e \in C$. A metric $d$ is defined on $\Gamma(G, C)$ by declaring each edge to be isometric to the unit interval. The graph $\Gamma(G, C)$ is $k$-almost convex if there exists an integer $N$ such that any two vertices $v_{1}$ and $v_{2}$ in $S(n)$ (the $n$-sphere centered at 1 ) with $d\left(v_{1}, v_{2}\right) \leq k$ can be joined by a path in $B(n)$ (the $n$-ball at 1 ) of length $\leq N$. For $k \geq 3$ it is an easy exercise to check that if $P$ is a finite presentation of a group $G$ (say with generating set $C)$ and $\Gamma(G, C)$ is $k$-almost convex, then if $X$ is the standard finite 2-complex corresponding to $P$ and $\tilde{X}$ is the universal cover of $X, \pi_{1}(\tilde{X}-D)$ is finitely generated for any finite subcomplex $D \subseteq \tilde{X}$. It follows from Tucker's theorem and our theorems 1 and 2 that if $M$ is a closed irreducible 3-manifold, $\pi_{1}(M)$ is infinite and for some generating set $C, \Gamma\left(\pi_{1}(M), C\right)$ is $k$-almost convex, $k \geq 3$, then the universal cover of $M$ is homeomorphic to $\mathbb{R}^{3}$. This is the main theorem of [9].

Theorem 3. If $G$ is a finitely presented group having a tame 1-combing, then $G$ is QSF.

The following class of groups is defined in [1].

Definition. If $X$ is a 1-complex and $\lambda, \epsilon>0$, a discrete path $p:\left[0, T_{p}\right] \cap \mathbb{N} \rightarrow$ $X^{0}$ is a $(\lambda, \epsilon)$-quasigeodesic if for any $x, y \in\left[0, T_{p}\right] \cap \mathbb{N}$ we have $\frac{1}{\lambda}|x-y|-\epsilon \leq$ $d(p(x), p(y)) \leq \lambda|x-y|+\epsilon$. A finitely presented group $G$ is in $C_{+}$if for some $\lambda, \epsilon>0, G$ has a bounded combing by $(\lambda, \epsilon)$-quasigeodesics. 
The following is a list of some of the groups in $C_{+}$: automatic groups, semihyperbolic groups, Coxeter groups, fundamental groups of closed 3-manifolds with everywhere non-positive sectional curvature, small cancellation groups, and any group which acts properly and cocompactly on a Tits building of Euclidean type (see [1]).

Theorem 4. If $G \in C_{+}$, then $G$ has a tame 1-combing.

Theorem 5. Asynchronously automatic groups have tame 1-combings.

Corollary 6. If $M$ is a closed irreducible 3-manifold with infinite fundamental group which is asynchronously automatic or in $C^{+}$, then the universal cover of $M$ is homeomorphic to $\mathbb{R}^{3}$.

In [8], some of our results have been extended to show that certain intermediate coverings of 3-manifolds are missing boundary manifolds. The results of [8] do not apply to all groups in $C^{+}$, but to an overlapping class of groups - those with almost prefix closed combings. The intermediate coverings considered are those corresponding to subgroups which are quasiconvex with respect to the almost prefix closed combing.

\section{Proofs of THEOREMS}

Proof of Theorem 1. It suffices to show that if the universal cover of $X$ has a tame 0 -combing or a tame 1-combing, then the universal cover of $Y$ does also, since the same argument will prove the converse. We do the tame 1-combing version, as the proof of the tame 0 -combing version is then easily seen. Fix vertices $*_{1}$ and $*_{2}$ of $X_{1}=X$ and $X_{2}=Y$ respectively. For $i \in\{1,2\}$, let $\left(\tilde{X}_{i}, \tilde{*}_{i}\right)$ be the universal cover of $\left(X_{i}, *_{i}\right)$ with covering projection $m_{i}$. The proof makes use of the following two facts basic to understanding the relationship between finite 2-complexes with the same fundamental group.

Lemma 1.1. If $\left(X_{1}, *_{1}\right)$ and $\left(X_{2}, *_{2}\right)$ are finite 2-complexes with isomorphic fundamental groups, then there are cellular maps $f_{1}:\left(X_{1}, *_{1}\right) \rightarrow\left(X_{2}, *_{2}\right)$ and $f_{2}$ : $\left(X_{2}, *_{2}\right) \rightarrow\left(X_{1}, *_{1}\right)$ such that $f_{2} \circ f_{1}$ and $f_{1} \circ f_{2}$ induce the identity on $\pi_{1}\left(X_{1}, *_{1}\right)$ and $\pi_{1}\left(X_{2}, *_{2}\right)$ respectively.

Lemma 1.2. Assume $f_{1}:\left(X_{1}, *_{1}\right) \rightarrow\left(X_{2}, *_{2}\right)$ and $f_{2}:\left(X_{2}, *_{2}\right) \rightarrow\left(X_{1}, *_{1}\right)$ are cellular maps such that $f_{1} \circ f_{2}$ induces the identity on $\pi_{1}\left(X_{2}, *_{2}\right)$. Let $\tilde{f}_{1}$ be the lift of $f_{1}$ to $\left(\tilde{X}_{1}, \tilde{*}_{1}\right)$ taking $\tilde{*}_{1}$ to $\tilde{*}_{2}$, and let $\tilde{f}_{2}$ be the lift of $f_{2}$ to $\left(\tilde{X}_{2}, \tilde{*}_{2}\right)$ taking $\tilde{*}_{2}$ to $\tilde{*}_{1}$ (see Figure 1). Then there exists an integer $N$ such that, for all $x \in \tilde{X}_{2}$, $\tilde{f}_{1} \circ \tilde{f}_{2}(x) \in S t^{N}(x)$.

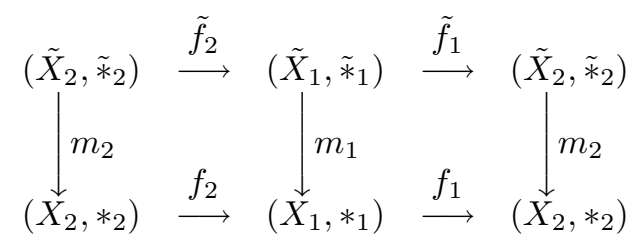

FiguRE 1 


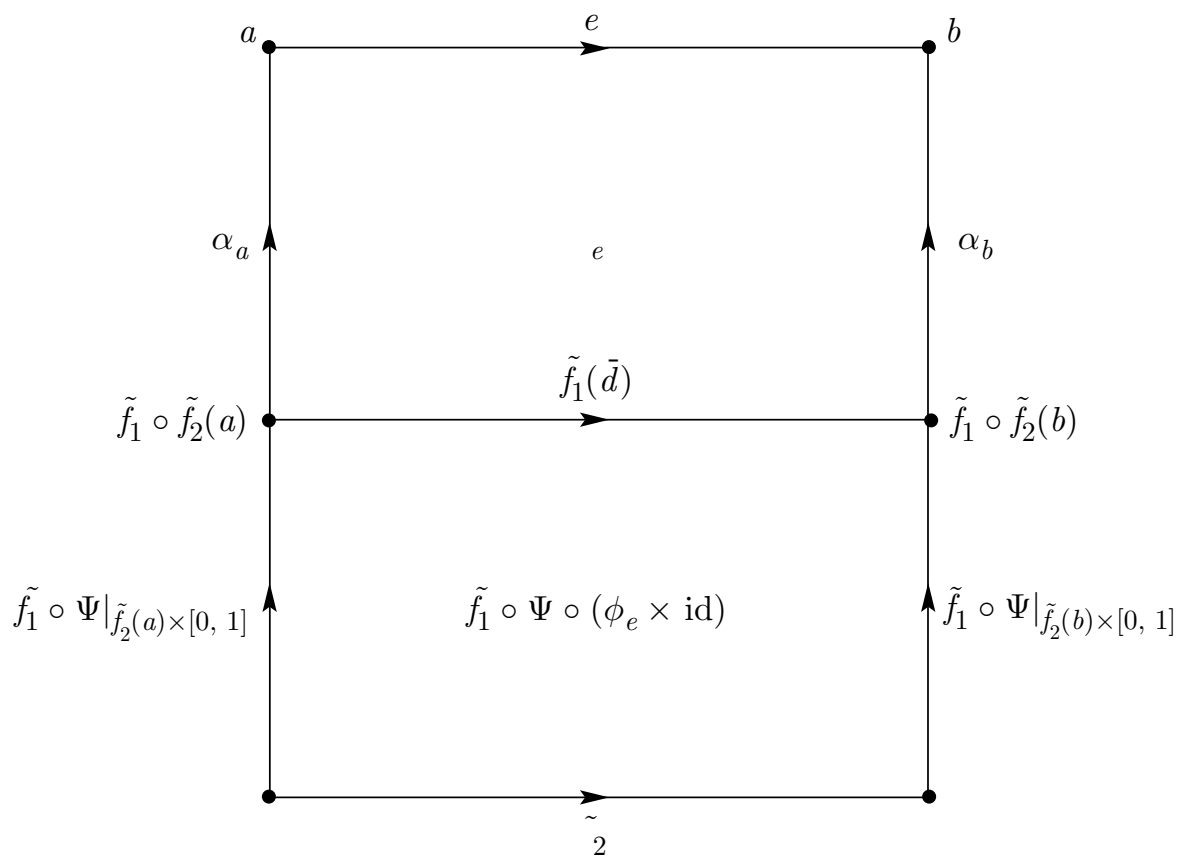

FiguRE 2

Given these two lemmas, to complete the proof of Theorem 1, take $f_{1}:\left(X_{1}, *_{1}\right) \rightarrow$ $\left(X_{2}, *_{2}\right)$ and $f_{2}:\left(X_{2}, *_{2}\right) \rightarrow\left(X_{1}, *_{1}\right)$ such that $f_{1} \circ f_{2}$ induces the identity on $\pi_{1}\left(X_{2}, *_{2}\right)$, and $f_{2} \circ f_{1}$ induces the identity on $\pi_{1}\left(X_{1}, *_{1}\right)$ by Lemma 1.1. For $i=1,2$, let $\tilde{f}_{i}$ be the lift of $f_{i}$ as in Lemma 1.2, and take $N$ to be sufficiently large so that, for all $x \in \tilde{X}_{2}, \tilde{f}_{1} \circ \tilde{f}_{2}(x) \in \mathrm{St}^{N}(x)$. For each vertex $v$ of $\tilde{X}_{2}$, let $\alpha_{v}$ be an edge path from $\tilde{f}_{1} \circ \tilde{f}_{2}(v)$ to $v$, of length $\leq N$.

Let $\Psi$ be a tame 1-combing for $\tilde{X}_{1}$, say, for the sake of simplicity and without loss of generality, with $\Psi(x, 0)=\tilde{*}_{1}$. Define a 0 -combing $\hat{\Psi}$ of $\tilde{X}_{2}$ and then extend this map to a 1-combing as follows. For each vertex $v$ of $\tilde{X}_{2}$ define $\hat{\Psi}$ on $\{v\} \times$ $[0,1]$ to be the path $\left.\tilde{f}_{1} \circ \Psi\right|_{\left\{\tilde{f}_{2}(v)\right\} \times[0,1]}$ followed by the path $\alpha_{v}$. Suppose $e$ is an edge of $\tilde{X}_{2}$ with initial point $a$ and endpoint $b$. If $\tilde{f}_{2}(a) \neq \tilde{f}_{2}(b)$, choose a simple edge path $\bar{d}=\left\langle d_{1}, d_{2}, \ldots, d_{n}\right\rangle$ in $\tilde{X}_{1}$ from $\tilde{f}_{2}(a)$ to $\tilde{f}_{2}(b)$, contained in $\tilde{f}_{2}(e)$, and homeomorphic to $e$ by $\phi_{e}: e \rightarrow \bar{d}$, and otherwise take $\bar{d}$ a constant path at $\tilde{f}_{2}(a)$, so $\phi_{e}: e \rightarrow \bar{d}$ is a constant map. Then $\tilde{f}_{1}(\bar{d})$ is a path from $\alpha_{a}(0)$ to $\alpha_{b}(0)$, with image in $\tilde{f}_{1} \circ \tilde{f}_{2}(e) \subseteq \mathrm{St}^{N}(e) \subseteq \mathrm{St}^{N+1}(a)$. There exists a fixed $M$ such that if $v$ is any vertex of $\tilde{X}_{2}$ and $\beta$ is a loop in $\operatorname{St}^{N+1}(v)$, then $\beta$ is homotopically trivial in $\mathrm{St}^{M}(v)$. Take a homotopy $H_{e}$ killing the loop $\left\langle\alpha_{a}^{-1}, \tilde{f}_{1}(\bar{d}), \alpha_{b}, e^{-1}\right\rangle$ in $\operatorname{St}^{M}(a)$ and define $\left.\hat{\Psi}\right|_{e \times[0,1]}$ by patching $H_{e}$ to $\tilde{f}_{1} \circ \Psi \circ\left(\phi_{e} \times\right.$ id $)$ as in Figure 2 .

To see that $\hat{\Psi}$ is tame, let $C$ be any compact subset of $\tilde{X}_{2}$. Then $C_{1}=\tilde{f}_{1}^{-1}(C) \cup$ $\left\{\tilde{*}_{1}\right\}$ is compact in $\tilde{X}_{1}$, since $\tilde{f}_{1}$ is proper (see [7]). Since $\Psi$ is tame, there exists a compact $D_{1} \subseteq \tilde{X}_{1}$ such that, if $c$ is any edge or vertex of $\tilde{X}_{1}$, then $\Psi^{-1}\left(C_{1}\right) \cap(c \times$ $[0,1])$ is contained in one component of $\Psi^{-1}\left(D_{1}\right) \cap(c \times[0,1])$. There are only finitely many edges $e$ of $\tilde{X}_{2}$ such that $\operatorname{im}\left(H_{e}\right) \cap C \neq \emptyset\left(\right.$ since $\operatorname{im}\left(H_{e}\right) \subseteq \mathrm{St}^{M}(a)$, where $a$ is the 
initial point of $e)$. Take $D$ to be the union of $\tilde{f}_{1}\left(D_{1}\right)$ and the $\operatorname{im}\left(\left.\hat{\Psi}\right|_{e \times[0,1]}\right)$ for these finitely many $e$. Then $D$ is compact, and for edges $e$ with $\operatorname{im}\left(H_{e}\right) \cap C \neq \emptyset$ we trivially have $\hat{\Psi}^{-1}(C) \cap(e \times[0,1])$ contained in one component of $\hat{\Psi}^{-1}(D) \cap(e \times[0,1])=$ $e \times[0,1] \operatorname{since} \operatorname{im}\left(\left.\hat{\Psi}\right|_{e \times[0,1]}\right) \subseteq D$. For $e$ with $\operatorname{im}\left(H_{e}\right) \cap C=\emptyset$ we need only check that $\left(\tilde{f}_{1} \circ \Psi \circ\left(\phi_{e} \times \mathrm{id}\right)\right)^{-1}(C)$ is contained in one component of $\left(\tilde{f}_{1} \circ \Psi \circ\left(\phi_{e} \times \mathrm{id}\right)\right)^{-1}(D)$. With $\operatorname{im}\left(\phi_{e}\right)=\bar{d}=\left\langle d_{1}, \ldots, d_{n}\right\rangle$ as before, for each $d_{i}$ by the choice of $D_{1}$, we have that $\Psi^{-1}\left(C_{1}\right) \cap\left(d_{i} \times[0,1]\right)$ is contained in one component of $\Psi^{-1}\left(D_{1}\right) \cap\left(d_{i} \times[0,1]\right)$. But $\tilde{*}_{1} \in C_{1}$ so $\Psi\left(d_{i} \times\{0\}\right)=\tilde{*}_{1} \in D_{1}$ and the one component of $\Psi^{-1}\left(D_{1}\right) \cap$ $\left(d_{i} \times[0,1]\right)$ of interest contains $d_{i} \times\{0\}$. Thus $\Psi^{-1}\left(C_{1}\right) \cap(\bar{d} \times[0,1])$ is contained in one component of $\Psi^{-1}\left(D_{1}\right) \cap(\bar{d} \times[0,1])$. Since $\tilde{f}_{1}^{-1}(C) \subseteq C_{1}$ and $\tilde{f}_{1}\left(D_{1}\right) \subseteq D$, we have

$$
\left(\tilde{f}_{1} \circ \Psi \circ\left(\phi_{e} \times \mathrm{id}\right)\right)^{-1}(C) \subseteq\left(\phi_{e} \times \mathrm{id}\right)^{-1}\left(\Psi^{-1}\left(C_{1}\right) \cap(\bar{d} \times[0,1])\right)
$$

is contained in one component of

$$
\left(\phi_{e} \times \mathrm{id}\right)^{-1}\left(\Psi^{-1}\left(D_{1}\right) \cap(\bar{d} \times[0,1])\right) \subseteq\left(\tilde{f}_{1} \circ \Psi \circ\left(\phi_{e} \times \mathrm{id}\right)\right)^{-1}(D)
$$

as required (and this also works if $\bar{d}$ is simply a constant path, since $D_{1}$ also witnesses that $\left.\Psi\right|_{X_{1}^{0} \times[0,1]}$ is tame). A similar but simpler argument applies to show that the restriction of $\hat{\Psi}$ to $\tilde{X}_{2}^{0} \times[0,1]$ is a tame 0 -combing, hence $\hat{\Psi}$ is tame.

Remark on Theorem 2. The spaces of this theorem are polyhedra rather than $\mathrm{CW}$ complexes. The reason for this is that if $A$ is a finite polyhedron and $B$ is a subcomplex of $A$, then $\pi_{1}(A-B)$ is finitely generated. The corresponding result is not true for $A$ a $\mathrm{CW}$-complex. R. Geoghegan has exhibited an elementary example of a finite CW-complex $A$ with one vertex $v$ such that $\pi_{1}(A-\{v\})$ is not finitely generated.

Proof of Theorem 2. First suppose that, for each finite subcomplex $C \subseteq \tilde{X}$, the group $\pi_{1}(\tilde{X}-C)$ is finitely generated. Define a nested sequence $C_{0} \subseteq C_{1} \subseteq C_{2} \subseteq \ldots$ of finite connected subcomplexes of $\tilde{X}$ whose union is $\tilde{X}$ as follows. Let $C_{0}=\emptyset$, and take $C_{1}$ to be a finite connected subcomplex of $\tilde{X}$ containing the vertex $*$ such that $\tilde{X}-C_{1}$ is a union of unbounded path components (where a set is unbounded in $\tilde{X}$ if it is contained in no compact subset of $\tilde{X})$. For $i \geq 2$, take $C_{i}$ to be a connected finite subcomplex of $\tilde{X}$ such that

$\left.a_{i}\right) C_{i}$ contains $\operatorname{St}\left(C_{i-1}\right)$,

$b_{i}$ ) each path component of $\tilde{X}-C_{i}$ is unbounded,

$c_{i}$ ) if $\Gamma$ is a path component of $\tilde{X}-C_{i-1}$, then $\Gamma \cap C_{i}$ is path connected and $C_{i}$ contains loops representing generators of some finite generating set of $\pi_{1}(\Gamma)$, and

$d_{i}$ ) if $i=2$, then $C_{2}$ is such that any loop in $C_{1}$ is homotopically trivial in $C_{2}$, and for $i \geq 3$, any loop based in $C_{i-2}$ and contained in $C_{i-1}-C_{i-3}$ is homotopic $\operatorname{rel}\{0,1\}$ in $C_{i}-C_{i-3}$ to a loop in $C_{i-2}-C_{i-3}$.

(To see this, construct the $C_{i}$ recursively. Take $C^{\prime}$ containing $\operatorname{St}\left(C_{1}\right)$ such that any loop in $C_{1}$ is homotopically trivial in $C^{\prime}$, add to $C^{\prime}$ enough paths to make $c_{2}$ ) hold for $C^{\prime}$, and then throw in any bounded path components of the complement of that to get $C_{2}$. Suppose $i \geq 3$ and that, for $j<i, C_{j}$ is a finite connected subcomplex of $\tilde{X}$ satisfying $\left.a_{j}\right)-d_{j}$ ). If $D$ is a connected finite subcomplex of $\tilde{X}$ satisfying $a_{i}$ ) or $d_{i}$ ) and $E$ is a finite connected subcomplex of $\tilde{X}$ containing $D$, then $E$ trivially satisfies $a_{i}$ ) or $d_{i}$ ) respectively. If $D$ is a connected finite subcomplex of 
$\tilde{X}$ containing $\operatorname{St}\left(C_{i-1}\right)$ and satisfying $\left.c_{i}\right)$, and $E$ is a finite connected subcomplex of $\tilde{X}$ containing $D$, then an elementary path connectedness argument shows that for $\Gamma$ as in $\left.c_{i}\right), \Gamma \cap E$ is path connected. Also $E$ trivially satisfies the second condition of $c_{i}$ ). Hence if we find $D_{a}, D_{c}$, and $D_{d}$ satisfying $\left.\left.a_{i}\right), c_{i}\right)$, and $d_{i}$ ) respectively, then the union of $D_{a}, D_{c}, D_{d}$ and all bounded path components of $\tilde{X}-\left(D_{a} \cup D_{c} \cup D_{d}\right)$ will be a connected finite subcomplex of $\tilde{X}$ satisfying $a(i)-d(i)$. Let $D_{a}$ be $\operatorname{St}\left(C_{i-1}\right)$. Let $D_{c}^{\prime}$ be the union of $\operatorname{St}\left(C_{i-1}\right)$ and, for each path component $\Gamma$ of $\tilde{X}-C_{i-1}$ (there are only finitely many), finitely many loops based in $\operatorname{St}\left(C_{i-1}\right)-C_{i-1}$ representing generators of some finite generating set of $\pi_{1}(\Gamma)$, together with paths in $\Gamma$ joining any two components of $\Gamma \cap \operatorname{St}\left(C_{i-1}\right)$. As $D_{c}^{\prime}$ is compact, there is a finite connected subcomplex $D_{c}$ of $\tilde{X}$ containing $D_{c}^{\prime}$. For each path component $\Gamma$ of $\tilde{X}-C_{i-3}, \pi_{1}(\Gamma)$ is finitely generated and, by $c_{i-2}$, any loop based in $C_{i-2}$ representing a generator of $\pi_{1}(\Gamma)$ is homotopic $\operatorname{rel}\{0,1\}$ in $\tilde{X}-C_{i-3}$ to a loop in $C_{i-2}-C_{i-3}$. Take a finite subcomplex $C^{\prime}$ containing $\operatorname{St}\left(C_{i-1}\right)$ and large enough to contain homotopies showing that for each such $\Gamma$, each loop based in $C_{i-2}$ in a finite generating set of $\pi_{1}\left(\Gamma \cap C_{i-1}\right)$ is homotopic rel $\{0,1\}$ to a loop in $C_{i-2}-C_{i-3}$. Then any loop based in $C_{i-2}$ and contained in $C_{i-1}-C_{i-3}$ is homotopic rel $\{0,1\}$ in $C^{\prime}-C_{i-3}$ to a loop in $C_{i-2}-C_{i-3}$.)

For each vertex $v \in \tilde{X}$, fix $\alpha_{v}$ to be an edge path from $*$ to $v$ such that, for all $i, \alpha_{v}^{-1}\left(C_{i}\right)$ is connected (since for each $i>0$ and each component $\Gamma$ of $\tilde{X}-C_{i-1}$, $\Gamma \cap C_{i}$ is path connected). Suppose $e$ is an edge of $\tilde{X}$ from a vertex $x$ to a vertex $y$. Let $k$ be the first integer such that $e \subseteq C_{k}$. For $0<i<k$, take $t_{i}, s_{i} \in[0,1]$ such that $\alpha_{x}^{-1}\left(C_{i}\right)=\left[0, t_{i}\right]$ and $\alpha_{y}^{-1}\left(C_{i}\right)=\left[0, s_{i}\right]$ (note that, by $a$ ), the $\left\{t_{i}\right\}$ and $\left\{s_{i}\right\}$ sequences are strictly increasing) and take $\beta(e, i)$ to be a path in $C_{i}-C_{i-1}$ from $\alpha_{x}\left(t_{i}\right)$ to $\alpha_{y}\left(s_{i}\right)$ (again since, for $\Gamma$ a path component of $\tilde{X}-C_{i-1}, \Gamma \cap C_{i}$ is path connected). The loop

$$
\left\langle\left.\alpha_{x}\right|_{\left[t_{k-1}, 1\right]}, e,\left.\alpha_{y}^{-1}\right|_{\left[s_{k-1}, 1\right]}, \beta^{-1}(e, k-1)\right\rangle
$$

has image in $C_{k}-C_{k-2}$ and hence is homotopic rel\{0,1\} to a loop $\gamma(e, k-1)$ in $C_{k-1}-C_{k-2}$ by a homotopy $H(e, k-1)$ in $C_{k+1}-C_{k-2}$ (by $\left.d\right)$ above). Suppose $1<i<k$ and we have defined a loop $\gamma(e, i)$ in $C_{i}-C_{i-1}$ based at $\alpha_{x}\left(t_{i}\right)$. Then $\left\langle\left.\alpha_{x}\right|_{\left[t_{i}, t_{i-1}\right]}, \gamma(e, i), \beta(e, i),\left.\alpha_{y}^{-1}\right|_{\left[s_{i}, s_{i-1}\right]}, \beta^{-1}(e, i-1)\right\rangle$ is a loop in $C_{i}-C_{i-2}$ and therefore is homotopic rel $\{0,1\}$ to a loop $\gamma(e, i-1)$ in $C_{i-1}-C_{i-2}$ by a homotopy $H(e, i-1)$ in $C_{i+1}-C_{i-2}$ (again, by $\left.d\right)$ above). Continuing until $\gamma(e, 1)$ and $H(e, 1)$ are defined, at the final step $\left\langle\left.\alpha_{x}\right|_{\left[0, t_{1}\right]}, \gamma(e, 1), \beta(e, 1),\left.\alpha_{y}^{-1}\right|_{\left[0, s_{1}\right]}\right\rangle$ is a loop in $C_{1}$, and therefore is homotopically trivial by a homotopy $H(e, 0)$ in $C_{2}$.

Define a 1-combing $\Psi$ of $\tilde{X}$ by defining, for each edge $e$ of $\tilde{X},\left.\Psi\right|_{e \times[0,1]}$ to be the homotopy combining the $H(e, i)$ for $i \in\{0,1, \ldots, k-1\}$ as in Figure 3 (clearly $\left.\Psi\right|_{\tilde{X} \times\{0\}}$ is constant and $\left.\Psi\right|_{\tilde{X} \times\{1\}}$ is the identity, and this is continuous since on the overlaps of these the $\left.\Psi\right|_{\{v\} \times[0,1]}$ are the fixed paths $\alpha_{v}$ ). Given any compact set $C$ in $\tilde{X}$, there exists $n$ sufficiently large so that $C \subseteq C_{n}$. Given any edge $e$ of $\tilde{X}$, for each $i$ such that $H(e, i)$ is defined, $H(e, i)$ has image in $C_{i+2}-C_{i-1}$. Hence if $i>n$, then $H(e, i) \cap C_{n}=\emptyset$, and so $\left.\Psi^{-1}\left(C_{n}\right) \cap(e \times[0,1])\right)$ is contained in the union of the domains of $H(e, 0), \ldots, H(e, n)$. As $\bigcup_{i=0}^{n} i m(H(e, i)) \subseteq C_{n+2}$, $\Psi^{-1}(C) \cap(e \times[0,1]) \subseteq \Psi^{-1}\left(C_{n}\right) \cap(e \times[0,1])$ is contained in one component of $\Psi^{-1}\left(C_{n+2}\right) \cap(e \times[0,1])$. By similar, but simpler reasoning, the restriction of $\Psi$ to $\tilde{X}^{0} \times[0,1]$ is a tame 0 -combing; hence $\Psi$ is a tame 1 -combing of $\tilde{X}$. 


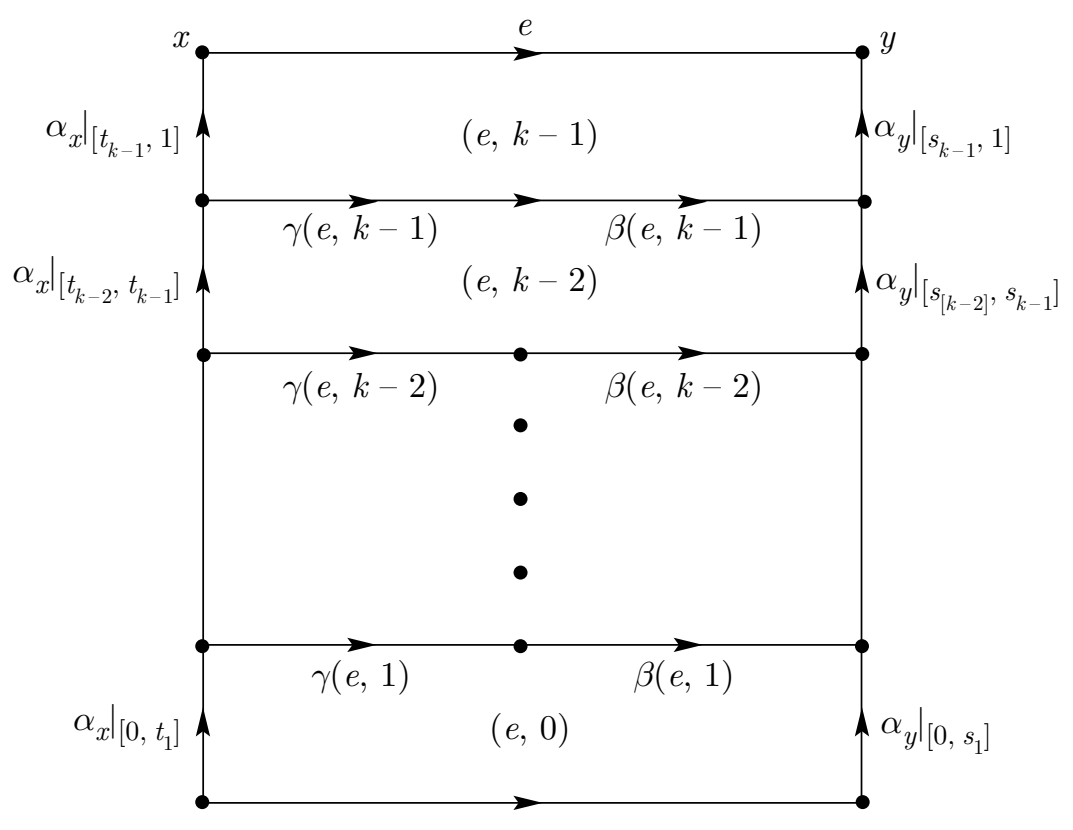

FIGURE 3

Conversely, suppose $\Psi$ is a tame 1-combing of $\tilde{X}$ and let $*=\Psi(\tilde{X} \times\{0\})$. Given any finite subcomplex $C$ of $\tilde{X}$, let $\Gamma$ be a component of $\tilde{X}-C$. If $\Gamma$ is bounded, then $\pi_{1}(\Gamma)$ is finitely generated. Otherwise, let $*^{\prime} \in \Gamma$ be a base point. Let $D$ be a finite subcomplex of $\tilde{X}$ containing $\left\{*, *^{\prime}\right\} \cup \operatorname{St}(C)$, and such that for any vertex or edge $c$ of $\tilde{X}, \Psi^{-1}(\operatorname{St}(C) \cup\{*\}) \cap(c \times[0,1])$ is contained in one path component of $\Psi^{-1}(D) \cap(c \times[0,1])$. Let $E$ be compact in $\tilde{X}$ such that for any vertex or edge $c$ of $\tilde{X}$, $\Psi^{-1}(\operatorname{St}(D)) \cap(c \times[0,1])$ is contained in one path component of $\Psi^{-1}(E) \cap(c \times[0,1])$.

Let $e$ be an edge of $\Gamma-D$ and let $T=e \times[0,1]$. Say that $\hat{D}$ and $\hat{E}$ are the path components of $\Psi^{-1}(D) \cap T$ and $\Psi^{-1}(E) \cap T$ containing $\Psi^{-1}(\operatorname{St}(C) \cup\{*\}) \cap T$ and $\Psi^{-1}(\operatorname{St}(D)) \cap T$ respectively. Clearly, $\hat{D} \cap(e \times\{1\})=\emptyset$. Let $a$ and $b$ be the initial point and endpoint of $e$.

Lemma 2.1. There is an arc $\beta$ in $T$ such that $\operatorname{im}(\beta) \cap(\{a\} \times[0,1])=\{(a, s)\}$ is the initial point of $\beta, \operatorname{im}(\beta) \cap(\{b\} \times[0,1])=\{(b, t)\}$ is the endpoint of $\beta, \beta$ separates $e \times\{1\}$ from $\Psi^{-1}(C) \cap T, i m(\beta) \cap \hat{D}=\emptyset$, and $(\{a\} \times[0, s]) \cup(\{b\} \times[0, t]) \cup i m(\beta) \subseteq \hat{E}$.

Proof. The map $\left.\Psi\right|_{T}$ is a uniformly continuous map of a rectangle into $\tilde{X}$. Choose $\epsilon_{1}>0$ such that for $x, y \in T$ and $d(x, y)<\epsilon_{1}, \Psi(x) \in \operatorname{St}(\Psi(y))$. Choose $\epsilon>0$ such that $2 \epsilon \leq \epsilon_{1}$ and $2 \epsilon<d(e \times\{1\}, \hat{D})$.

If $x \in \operatorname{bd}(\hat{D}) \cap(\{a, b\} \times[0,1])$, then let $D_{x}$ be the disk of radius $\epsilon$ in $T$ centered at $x$. If $x \in \operatorname{bd}(\hat{D})-(\{a, b\} \times[0,1])$, let $D_{x}$ be a closed disk of radius less than the minimum of $\epsilon$ and $d(x,\{a, b\} \times[0,1])$, centered at $x$. By the compactness of $\operatorname{bd}(\hat{D})$, there are finitely many $D_{x}$, call them $D_{1}, \ldots, D_{n}$, in $T$ such that $\operatorname{bd}(\hat{D}) \subseteq$ $\bigcup_{i=1}^{n} \operatorname{int}\left(D_{i}\right)$. By slightly enlarging the radius of some $D_{i}$, we may assume that no two $D_{i}$ intersect at a single point, while retaining the properties that $\Psi\left(D_{i}\right) \subseteq$ 


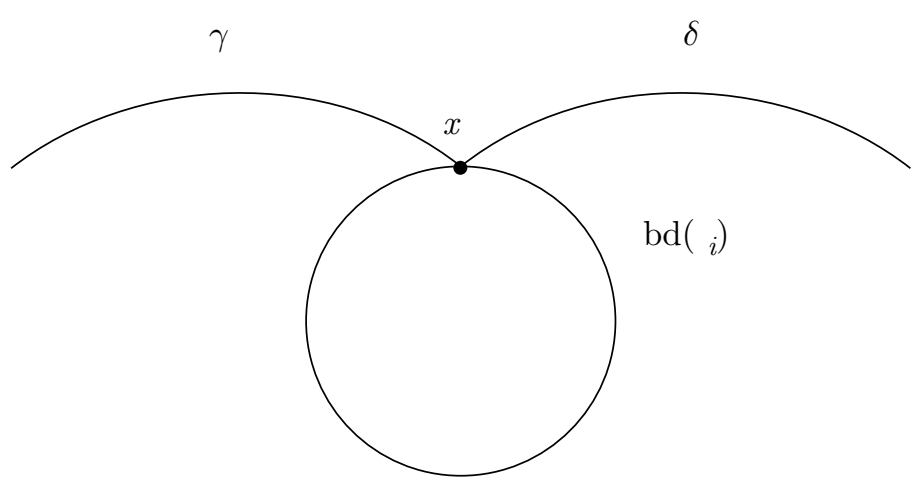

Figure 4

$\operatorname{St}(D)$, if $D_{i}$ is not centered at a point of $\{a, b\} \times[0,1]$ then $D_{i} \cap(\{a, b\} \times[0,1])=\emptyset$, and $\left(\bigcup_{i=1}^{n} D_{i}\right) \cap(e \times\{1\})=\emptyset$.

The boundary of $\bigcup_{i=1}^{n} D_{i}$ is a finite union of arcs of circles. Say that $\gamma$ and $\delta$ are two such arcs and $x \in \gamma \cap \delta$. If $\operatorname{bd}\left(D_{k}\right)$ is a circle containing $x$ but not containing $\gamma$ or $\delta$, then there are open arcs on either side of $x \operatorname{in} \operatorname{bd}\left(D_{k}\right)$ that are subsets of $\bigcup_{i=1}^{n}$ int $D_{i}$ (see Figure 4), i.e., each component of bd $\left(\bigcup_{i=1}^{n} D_{i}\right)$ is a 1-manifold.

Let $s$ be the largest number with $(a, s) \in \bigcup_{i=1}^{n} D_{i}$. Then $(a, s) \in \operatorname{bd}\left(\bigcup_{i=1}^{n} D_{i}\right)$, so $(a, s) \notin \hat{D}$. Let $\beta$ be the component of $\operatorname{bd}\left(\bigcup_{i=1}^{n} D_{i}\right)$ containing $(a, s)$. Observe that $\beta$ is an arc with initial point $(a, s)$ and, for some $k,(a, s) \in \operatorname{bd}\left(D_{k}\right)$, where $D_{k}$ is one of the disks centered at an $\left(a, s^{\prime}\right) \in \operatorname{bd}(\hat{D})$ with $s^{\prime}<s$. Also, $\beta$ does not intersect $\hat{D}\left(\operatorname{as} \operatorname{bd}(\hat{D}) \subseteq \operatorname{int}\left(\bigcup_{i=1}^{n} D_{i}\right)\right)$. If the endpoint of $\beta$ were in $\{a\} \times[0,1]$, then $\beta$ would separate $\operatorname{int}\left(D_{k}\right)$ from $(a, 0)$ and hence separate points of $\hat{D}$, contrary to the connectedness of $\hat{D}$. By the definition of $\epsilon, \operatorname{im}(\beta) \cap(e \times\{1\})=\emptyset$. Thus the endpoint of $\beta$ is in $\{b\} \times[0,1]$. Since $\{a\} \times\{0, s\} \subseteq \Psi^{-1}(\operatorname{St}(D)) \cap(\{a\} \times[0,1])$, we have $\{a\} \times[0, s] \subseteq \hat{E}$. Similarly, if $(b, t)$ is the terminal point of $\beta$, then $\{b\} \times[0, t] \subseteq \hat{E}$. Since $\Psi\left(\bigcup_{i=1}^{n} D_{i}\right) \subseteq \operatorname{St}(D)$, we have $\operatorname{im}(\Psi \circ \beta) \subseteq \operatorname{St}(D)$ so $\operatorname{im}(\beta) \subseteq \hat{E}$. Now $\beta$ separates $e \times\{1\}$ from $(a, 0)$. But $\hat{D}$ is connected and $(a, 0) \in \hat{D}$, so $\beta$ separates $e \times\{1\}$ from $\hat{D}$ and hence $e \times\{1\}$ from $\Psi^{-1}(C)$.

Let $a$ be a vertex of $\Gamma$. Define $\ell(a) \in[0,1]$ to be the largest number such that $\Psi(\{a\} \times[0, \ell(a)]) \subseteq D$. Now $\Psi^{-1}(\operatorname{St}(C)) \cap(\{a\} \times[0,1]) \subseteq[0, \ell(a)]$, and if $e$ is an edge of $\tilde{X}-D$ with initial vertex $a$, then the $s$ given in Lemma 2.1 is larger than $\ell(a)$. By Lemma 2.1, we have that if $e$ is an edge of $\Gamma-D$ with initial point $a$ and terminal point $b$, then $\Psi$ gives a homotopy between $e$ and

$$
\left\langle\left(\left.\Psi\right|_{\{a\} \times[s, 1]}\right)^{-1}, \Psi \circ \beta,\left.\Psi\right|_{\{b\} \times[t, 1]}\right\rangle
$$

(where $s$ and $t$ are defined in Lemma 2.1). Furthermore, the image of this homotopy does not intersect $C$, and so lies in $\Gamma$. Combining this homotopy with two homotopies that eliminate backtracking in $\{a\} \times[0,1]$ and $\{b\} \times[0,1]$, we have that $e$ is homotopic to

$$
\left\langle\left(\left.\Psi\right|_{\{a\} \times[\ell(a), 1]}\right)^{-1},\left.\Psi\right|_{\{a\} \times[\ell(a), s]}, \Psi \circ \beta,\left(\left.\Psi\right|_{\{b\} \times[\ell(b), t]}\right)^{-1},\left.\Psi\right|_{\{b\} \times[\ell(b), 1]}\right\rangle
$$

by a homotopy in $\Gamma$. By the choice of $\ell(a), \ell(b), a$, and $b, \operatorname{im}\left(\left.\Psi\right|_{\{a\} \times[\ell(a), s]}\right) \cup$ $\operatorname{im}\left(\left.\Psi\right|_{\{b\} \times[\ell(b), t]}\right)$ is a subset of $E-C$. Hence, if $e$ is an edge of $\Gamma-D$, with initial point 


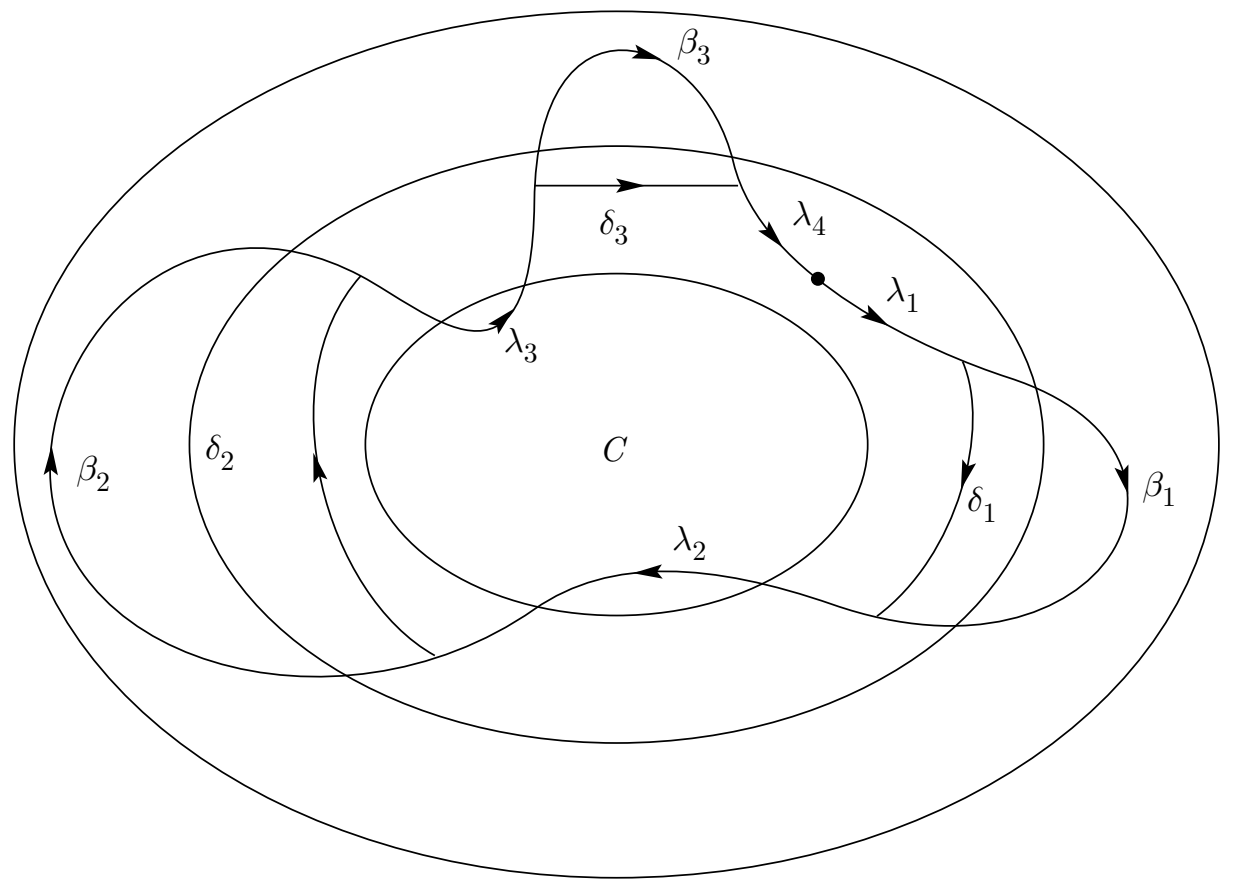

FiguRE 5

$a$ and endpoint $b$, then $e$ is homotopic to a path $\left\langle\left(\left.\Psi\right|_{\{a\} \times[\ell(a), 1]}\right)^{-1}, \alpha,\left.\Psi\right|_{\{b\} \times[\ell(b), 1]}\right\rangle$ by a homotopy $H_{e}$ in $\hat{\Gamma}$, where $\alpha$ is a path with $\operatorname{im}(\alpha) \subseteq E$.

Now let $\tau$ be any edge loop $\left\langle e_{1}, e_{2}, \ldots, e_{n}\right\rangle$ in $\Gamma$ based at $*^{\prime}$. If $e_{i}$ is an edge of $D$, let $H_{e_{i}}$ be the trivial homotopy of $e_{i}$ to itself. If $e_{i}$ is an edge of $\operatorname{St}(D)$ but not of $D$, then there are two cases when defining $H_{e_{i}}$. If both the initial vertex $a$ and the terminal vertex $b$ of $e_{i}$ lie in $\operatorname{St}(D)-D$, then let $H_{e_{i}}$ be the homotopy of $e_{i}$ to

$$
\left\langle\left(\left.\Psi\right|_{\{a\} \times[\ell(a), 1]}\right)^{-1},\left.\Psi\right|_{\{a\} \times[\ell(a), 1]}, e_{i},\left(\left.\Psi\right|_{\{b\} \times[\ell(b), 1]}\right)^{-1},\left.\Psi\right|_{\{b\} \times[\ell(b), 1]}\right\rangle
$$

that eliminates backtracking in $\{a\} \times[0,1]$ and $\{b\} \times[0,1]$. Similarly define $H_{e_{i}}$ if one vertex of $e_{i}$ is in $\operatorname{St}(D)-D$ and the other vertex is in $D$.

Combining the homotopies $H_{e_{i}}$ gives a homotopy of $\tau \operatorname{rel}\left\{*^{\prime}\right\}$ to a loop in $E \cap \Gamma$. As $\pi_{1}\left(E \cap \Gamma, *^{\prime}\right)$ is finitely generated, $\pi_{1}\left(\Gamma, *^{\prime}\right)$ is finitely generated.

Proof of Theorem 3. Take $X$ to be the standard 2-complex for a finite presentation of $G$ and $\tilde{X}$ to be the universal cover of $X$. Assume $G$ has a tame 1-combing, or equivalently, by the last theorem, for any finite subcomplex $C$ of $\tilde{X}, \pi_{1}(\tilde{X}-C)$ is finitely generated. (A proof that $G$ is QSF can be given using either of these. We give the version using the latter condition.) Let $C$ be a connected finite subcomplex of $\tilde{X}$. Choose $D$ a connected finite subcomplex of $\tilde{X}$ containing $\operatorname{St}(C)$ such that for each component $\Gamma$ of $\tilde{X}-C, D \cap \Gamma$ is connected and $D$ contains loops (based in $\Gamma \cap D$ ) representing generators in a finite generating set for $\pi_{1}(\Gamma)$. Let $E$ be a connected finite subcomplex of $X$ containing $D$ such that any loop in $D$ is homotopically trivial in $E$. Say that $\alpha_{1}, \ldots, \alpha_{n}$ are edge path loops based at $* \in C$ such that $\left[\alpha_{1}\right], \ldots,\left[\alpha_{n}\right]$ generate $\pi_{1}(E, *)$. 
If $\alpha \in\left\{\alpha_{1}, \ldots, \alpha_{n}\right\}$, then $\alpha$ can be written as $\left\langle\lambda_{1}, \beta_{1}, \lambda_{2}, \beta_{2}, \cdots, \lambda_{k-1}, \beta_{k-1}, \lambda_{k}\right\rangle$, where $\lambda_{i}$ is an edge path in $D$ and $\beta_{i}$ is an edge path in $E-C$. By the definition of $D$, there is an edge path $\delta_{i}$ in $D-C$ from $\beta_{i}(0)$ to $\beta_{i}(1)$ (see Figure 5$)$. Let

$$
\gamma_{i}=\left\langle\lambda_{1}, \delta_{1}, \ldots, \lambda_{i-1}, \delta_{i-1}, \lambda_{i}, \beta_{i}, \delta_{i}^{-1}, \lambda_{i}^{-1}, \delta_{i-1}^{-1}, \lambda_{i-1}^{-1}, \ldots, \delta_{1}^{-1}, \lambda_{1}^{-1}\right\rangle .
$$

Then $\alpha$ is homotopic $\operatorname{rel}\{0,1\}$ to $\left\langle\gamma_{1}, \ldots, \gamma_{k-1}, \xi\right\rangle$ by a homotopy in $E$, where $\xi=\left\langle\lambda_{1}, \delta_{1}, \ldots, \lambda_{k-1}, \delta_{k-1}, \lambda_{k}\right\rangle$. As $\xi$ is a loop in $D, \xi$ is homotopically trivial in $E$. The loop $\left\langle\beta_{i}, \delta_{i}^{-1}\right\rangle$ has image in $E-C$ with initial point $*^{\prime}$, a vertex of $D$. Let $\Gamma$ be the component of $\tilde{X}-C$ containing $*^{\prime}$ (and hence $\operatorname{im}\left\langle\beta_{i}, \delta_{i}^{-1}\right\rangle$ ). By the definition of $D$ there exist a point $*^{\prime \prime} \in \Gamma \cap D$ and finitely many loops in $D \cap \Gamma$, based at $*^{\prime \prime}$, which represent generators for $\pi_{1}\left(\Gamma, *^{\prime \prime}\right)$. As $D \cap \Gamma$ is connected, there are finitely many loops in $D \cap \Gamma$ based at $*^{\prime}$ which represent generators for $\pi_{1}\left(\Gamma, *^{\prime}\right)$. As $\left\langle\beta_{i}, \delta_{i}^{-1}\right\rangle$ is a loop in $\Gamma$ passing through $*^{\prime},\left\langle\beta_{i}, \delta_{i}^{-1}\right\rangle$ is homotopic $\operatorname{rel}\left\{*^{\prime}\right\}$ to a product of loops in $D \cap \Gamma$ based at $*^{\prime}$, by a homotopy in $\Gamma$. Hence there is a map $H_{i}$ of a 2-disk into $\Gamma$ such that $H_{i}$ restricted to the boundary of the disk is the loop $\left\langle\beta_{i}, \delta_{i}^{-1}\right\rangle$ followed by a loop in $D \cap \Gamma$.

Define a finite 2-complex $K$ and a map of $f: K \rightarrow \tilde{X}$ as follows. Take $K$ to be $E$ with a finite number of attached 2-disks, and take $\left.f\right|_{E}$ to be the inclusion map of $E$ into $\tilde{X}$. For each $\alpha \in\left\{\alpha_{1}, \ldots, \alpha_{n}\right\}$ and each corresponding $\left\langle\beta_{i}, \delta_{i}^{-1}\right\rangle$, attach the 2-disk $\operatorname{dom}\left(H_{i}\right)$ to $E$ with attaching map $H_{i}$ restricted to the boundary of $\operatorname{dom}\left(H_{i}\right)$. Take $f$ on this 2 -disk to be defined by $H_{i}$. Observe that in $K,\left\langle\beta_{i}, \delta_{i}^{-1}\right\rangle$ is homotopic to a loop in $D$, which by the definition of $E$ is homotopically trivial in $E$. In other words, all $\gamma_{i}$ for $i \in\{1, \ldots, k-1\}$ are homotopically trivial in $K$. Hence each $\alpha_{i}$ is homotopic in $K$ to a loop in $D$, and this loop is then homotopically trivial in $K$. As $\left[\alpha_{1}\right], \cdots,\left[\alpha_{n}\right]$ are generators of $\pi_{1}(K, *)$, we have that $K$ is simply connected. As $\operatorname{im}\left(H_{i}\right) \subseteq \tilde{X}-C$, we have that $\left.f\right|_{f^{-1}(C)}: f^{-1}(C) \rightarrow C$ is a homeomorphism. Hence $G$ is QSF.

Proof of Theorem 4. Suppose $G \in C_{+}$, let $X$ be the standard 2-complex for some finite presentation of $G$, and let $\tilde{X}$ be the universal cover of $X$. Take $\lambda$ and $\epsilon$ such that $\tilde{X}$ has a bounded combing by $(\lambda, \epsilon)$-quasigeodesics. First define a tame 0 -combing $\Psi$ on $\tilde{X}$ as follows. If $v$ is a vertex of $\tilde{X}$, then let $p_{v}:\left[0, T_{v}\right] \cap \mathbb{N}$ be the $(\lambda, \epsilon)$-quasigeodesic from a fixed $* \in \tilde{X}$ to $v$ in a bounded combing of $\tilde{X}$. Extend $p_{v}$ to $\left[0, T_{v}\right]$ by taking edges between successive vertices in the path and define $\left.\Psi\right|_{\{v\} \times[0,1]}$ by $\Psi(v, t)=p_{v}\left(t T_{v}\right)$ (a simple reparameterization of $p_{v}$ ). Since the $p_{v}$ are part of a bounded combing, $\Psi$ is a bounded 0 -combing (see the remarks after the definition of bounded 0-combing above).

To see that $\Psi$ is tame, it suffices to show that for any compact $C=\operatorname{St}^{N}(*) \subseteq \tilde{X}$, there exists a compact $D=\mathrm{St}^{M}(*) \subseteq \tilde{X}$ such that, for any vertex $v, \Psi^{-1}(C) \cap$ $(\{v\} \times[0,1])$ is contained in one component of $\Psi^{-1}(D) \cap(\{v\} \times[0,1])$. Let $M=$ $\lambda^{2} N+\lambda^{2} \epsilon+\epsilon$. Then since $\left.\Psi\right|_{\{v\} \times[0,1]}$ is simply a reparameterization of $p_{v}$, it suffices to show that if $0<t_{1}<t_{2} \leq T_{v}$ and $p_{v}\left(t_{1}\right) \in \tilde{X}-\mathrm{St}^{M}(*)$, then $p_{v}\left(t_{2}\right) \in \tilde{X}-\mathrm{St}^{N}(*)$. Suppose to the contrary that $d\left(*, p_{v}\left(t_{1}\right)\right)>M$ and $d\left(*, p_{v}\left(t_{2}\right)\right) \leq N$. Then $M<$ $\lambda t_{1}+\epsilon$ and $\frac{1}{\lambda} t_{2}-\epsilon \leq N$, since $p_{v}$ is a $(\lambda, \epsilon)$-quasigeodesic. But then

$$
\lambda t_{2}+\epsilon=\lambda^{2}\left(\frac{1}{\lambda} t_{2}-\epsilon\right)+\lambda^{2} \epsilon+\epsilon \leq \lambda^{2} N+\lambda^{2} \epsilon+\epsilon=M<\lambda t_{1}+\epsilon<\lambda t_{2}+\epsilon,
$$

a contradiction. 


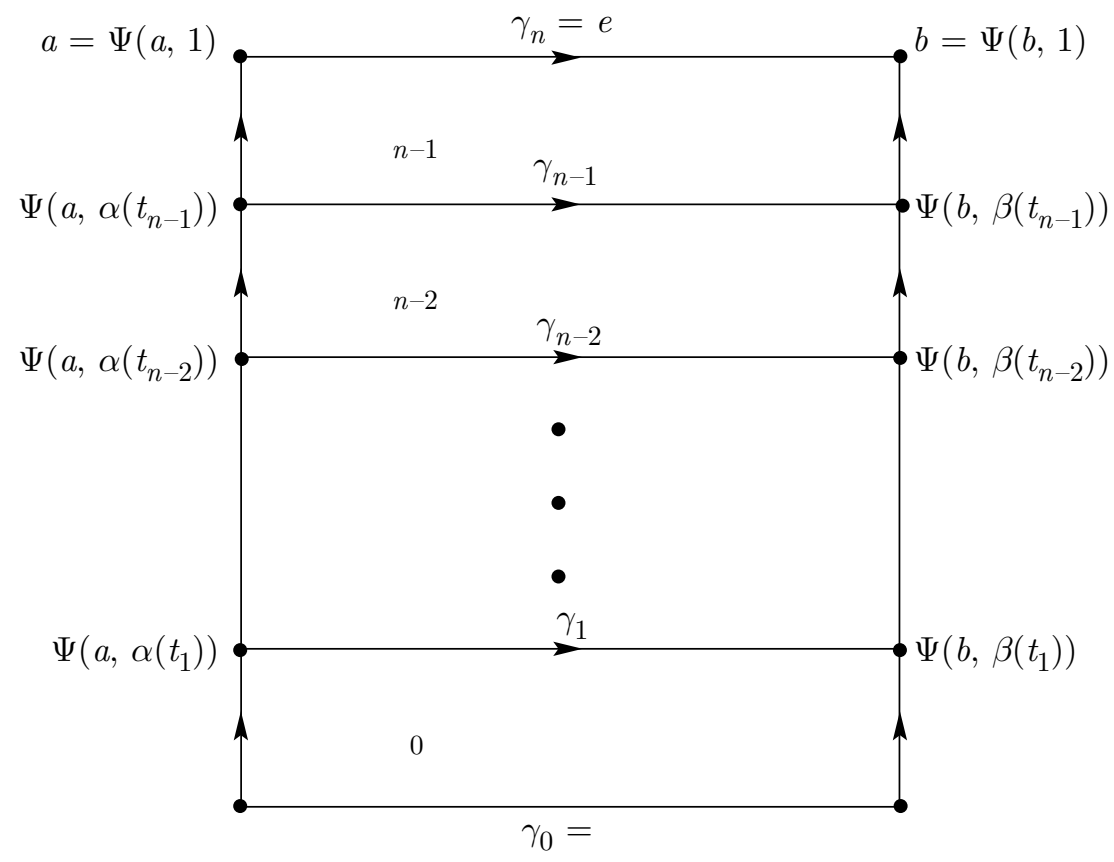

FiguRE 6

Thus there exists a bounded, tame 0 -combing of $\tilde{X}$. Theorem 4 is now an immediate consequence of the following lemma.

Lemma 4.1. Suppose $\tilde{X}$ is the universal cover of a finite 2-complex $X$. If $\tilde{X}$ has a bounded, tame 0-combing, then $\tilde{X}$ has a tame 1-combing.

Proof. Suppose $\Psi$ is a bounded, tame 0 -combing of $\tilde{X}$, and take $K$ to be a suitable constant in the definition of bounded 0 -combing. Let $e$ be an edge of $\tilde{X}$, with initial point $a$ and endpoint $b$. Take $\alpha, \beta:[0,1] \rightarrow[0,1]$ orientation preserving homeomorphisms such that $d(\Psi(a, \alpha(t)), \Psi(b, \beta(t)))<K$. Consider a partition of $[0,1], 0=t_{0}<t_{1}<\ldots<t_{n}=1$, such that, for all $i<n, \Psi\left(\{a\} \times\left.\alpha\right|_{\left[t_{i}, t_{i+1}\right]}\right) \subseteq$ $\operatorname{St}\left(\Psi\left(a, \alpha\left(t_{i}\right)\right)\right)$ and $\Psi\left(\{b\} \times\left.\beta\right|_{\left[t_{i}, t_{i+1}\right]}\right) \subseteq \operatorname{St}\left(\Psi\left(b, \beta\left(t_{i}\right)\right)\right)$. Then, for all $i<n$, since $d\left(\Psi\left(a, \alpha\left(t_{i}\right)\right), \Psi\left(b, \beta\left(t_{i}\right)\right)\right) \leq K$, there exists a path $\gamma_{i}$ from $\Psi\left(a, \alpha\left(t_{i}\right)\right)$ to $\Psi\left(b, \beta\left(t_{i}\right)\right)$ of length at most $K$. Let $\gamma_{n}=e$ and take $\gamma_{0}$ to be the constant map at $*$. For a fixed integer $L$, depending only on $K$, if $\gamma$ is a loop of length $\leq 2 K+2$ in $\tilde{X}$, then $\gamma$ is homotopically trivial, by a homotopy in $\operatorname{St}^{L}(v)$, for any $v$ in the image of the homotopy. Extend $\Psi$ to $e \times[0,1]$ by patching together homotopies $H_{i}$ as in Figure 6 , where $H_{i}$ is a homotopy killing $\left\langle\gamma_{i}, \Psi\left(\{b\} \times\left.\beta\right|_{\left[t_{i}, t_{i+1}\right]}\right), \gamma_{i+1}^{-1},\left(\Psi\left(\{a\} \times\left.\alpha\right|_{\left[t_{i-1}, t_{i}\right]}\right)\right)^{-1}\right\rangle$ in $\mathrm{St}^{L}\left(\Psi\left(a, \alpha\left(t_{i}\right)\right)\right)$.

Given a compact $C \subseteq \tilde{X}$, let $C^{\prime}=\operatorname{St}^{L}(C)$, and take $D^{\prime} \subseteq \tilde{X}$ to be a compact set such that, for any vertex $v$ of $\tilde{X}, \Psi^{-1}\left(\operatorname{St}^{L}(C)\right) \cap(\{v\} \times[0,1])$ is contained in one path component of $\Psi^{-1}\left(D^{\prime}\right) \cap(\{v\} \times[0,1])$. Let $D=\operatorname{St}^{L}\left(D^{\prime}\right)$. Suppose $e$ is an edge with initial point $a$ and endpoint $b$, and $\left.\Psi\right|_{e \times[0,1]}$ is defined by the $H_{i}$ as above. If $x \in \Psi^{-1}(C) \cap(e \times[0,1])$, then $x \in \operatorname{dom}\left(H_{i}\right)$ for an $i$ with $\Psi\left(a, \alpha\left(t_{i}\right)\right) \in \operatorname{St}^{L}\left(H_{i}(x)\right) \subseteq$ $\mathrm{St}^{L}(C)=C^{\prime}$. Thus $\left(a, \alpha\left(t_{i}\right)\right)$ is in the one path component of $\Psi^{-1}\left(D^{\prime}\right) \cap(\{a\} \times[0,1])$ 
containing $\Psi^{-1}\left(C^{\prime}\right) \cap(\{a\} \times[0,1])$. If $\Psi\left(a, \alpha\left(t_{i}\right)\right) \in D^{\prime}$, then, for all $y \in \operatorname{dom}\left(H_{i}\right)$, $H_{i}(y) \in \mathrm{St}^{L}\left(\Psi\left(a, \alpha\left(t_{i}\right)\right)\right) \in \mathrm{St}^{L}\left(D^{\prime}\right)=D ; \operatorname{so} \operatorname{im}\left(H_{i}\right) \subseteq D$. Thus $\Psi^{-1}(C) \cap(e \times[0,1])$ is contained in a union of the $\operatorname{dom}\left(H_{i}\right) \subseteq D$ for which $\left(a, \alpha\left(t_{i}\right)\right)$ is in a subinterval of $\{a\} \times[0,1]$ determined by a path component of $\Psi^{-1}\left(D^{\prime}\right) \cap(\{a\} \times[0,1])$; but then this union is contained in a one-path component of $\Psi^{-1}(D) \cap(e \times[0,1])$.

As we said above, this also completes the proof of Theorem 4 .

Proof of Theorem 5. By [6], $G$ has an asynchronously automatic structure with uniqueness, and the combing corresponding to this regular language is asynchronously bounded. Moreover, this combing will have a departure function $\Delta: \mathbb{R} \rightarrow \mathbb{R}$ such that for any word $w$ in the regular language, any $r, s \geq 0$, and any $t \geq \Delta(r)$ with $s+t$ less than the length of $w$, the distance in the Cayley graph of $G$ from $w(s)$ to $w(s+t)$ is greater than $r$ (see [6] or [4]). Given any compact $C$, taking $r$ sufficiently large so $C \subseteq \mathrm{St}^{r}(*)$, and taking $D=\mathrm{St}^{\Delta(r)}(*)$, we see that a combing with a departure function corresponds to a tame 0-combing (but the converse need not hold). Passing to the continuous combing corresponding to the combing from the asynchronous structure of $G$, we get that $G$ has a bounded, tame 0-combing. By Lemma 4.1, this 0-combing can then be extended to a tame 1-combing.

The use of a departure function in the above argument simplifies our original proof of this result. In fact, the proof that the 0-combing derived from an asynchronus automatic structure of a group is tame, is somewhat easier than the proof that there is a departure function, but there seems little reason to include the details here. Automatic groups are included in the class of asynchronously automatic groups and also belong to $C_{+}$; hence we have two ways of showing that such groups have asynchronously bounded, tame 0 -combings.

\section{ConCluding REMARKS}

Suppose $X$ is a finite 2-complex with fundamental group $G$ and universal cover $\tilde{X}$. In the search for the definition of tame 1-combing, a (most likely strictly) weaker combing condition on $\tilde{X}$ was discovered that also implies $G$ is QSF, namely that there exists a 1-combing $\Psi$ of $\tilde{X}$ such that, for any compact $C \subseteq \tilde{X}$, there exists a compact $D \subseteq \tilde{X}$ with $\Psi^{-1}(C)$ contained in one component of $\Psi^{-1}(D)$. The definition of tame 1-combing corresponds to a sort of uniform localization of this property. While this property is simpler to state, the 1-combings we construct are all tame, so there seems little reason at this time to develop this idea further.

Suppose $\Psi$ is a 1-combing of $\tilde{X}$ such that, for all compact $C \subseteq \tilde{X}$, there exists a compact $D \subseteq \tilde{X}$ such that, for any edge $e$ of $\tilde{X}, \Psi^{-1}(C) \cap(e \times[0,1])$ is contained in one path component of $\Psi^{-1}(D) \cap(e \times[0,1])$ (i.e., part of the definition of tame 1-combing, but dropping the condition that the restriction of $\Psi$ to $\tilde{X}^{0}$ is a tame 0 -combing). Then (with some effort) it can be shown that there must also exist a tame 1-combing of $\tilde{X}$. Again, there seems little reason to pursue this definition since the most convenient way of constructing a tame 1-combing has been to first construct a tame 0-combing which can be extended.

In terms of pro-groups, the condition that for each finite subcomplex $C$ of $\tilde{X}$, $\pi_{1}(\tilde{X}-C)$ is finitely generated, is equivalent to the condition that for each end $\mathcal{E}$ of $\tilde{X}$, pro- $\pi_{1}(\mathcal{E})$ is pro-finitely generated. 


\section{REFERENCES}

1. J. M. Alonso and M. R. Bridson, Semihyperbolic groups, Proc. London Math. Soc. 70, Part I (1995), 56-114. MR 95j:20033

2. M. Bestvina and G. Mess, The boundary of negatively curved groups, JAMS 4 (1991), 469-481. MR 93j:20076

3. S. G. Brick and M. L. Mihalik, The QSF property for groups and spaces, Math. Z. 220 (1995), 202-217. MR 96i:57009

4. M. Bridson, Combings of semidirect products and 3-manifold groups, Geometric and Functional Analysis 3, No. 3 (1993), 263-278. MR 94i:20065

5. M. G. Brin and T. L. Thickstun, 3-manifolds which are end 1-movable, Memoirs of the American Math. Soc. 81, No. 411 (1989). MR 90g:57015

6. D. B. A. Epstein, J. W. Cannon, D. F. Holt, S. V. F. Levy, M. S. Paterson, and W. P. Thurston, Word processing in groups, Jones and Bartlett, Boston and London, 1992. MR 93i:20036

7. M. L. Mihalik, Ends of fundamental groups in shape and proper homotopy, Pacific J. of Math. 88, No. 2 (1980), 431-458. MR 82g:55014

8. M. L. Mihalik, Compactifying coverings of 3-manifolds, Comment. Math. Helv. 71 (1996), 362-372. CMP 97:04

9. V. Poénaru, Almost convex groups, Lipschitz combing, and $\pi_{1}^{\infty}$ for universal covering spaces of closed 3-manifolds, J. Diff. Geom. 35 (1992), 103-130. MR 93d:57032

10. T. W. Tucker, Non-compact 3-manifolds and the missing boundary problem, Topology 13 (1974), 267-273. MR 50:5801

Department of Mathematics, Vanderbilt University, Nashville, Tennessee 37240

E-mail address: mihalikm@ctrvax.vanderbilt.edu

E-mail address: tschantz@athena.cas.vanderbilt.edu 